\title{
Numerical Study of Smoke Extraction for Adhered Spill Plumes in Atria: Impact of Extraction Rate and Geometrical Parameters
}

\author{
Nele Tilley and Bart Merci \\ Ghent University, Department of Flow, Heat and Combustion Mechanics, \\ Sint-Pietersnieuwstraat 41, B-9000 Ghent, Belgium \\ Corr. author: Bart.Merci@UGent.be, Tel: +32 926433 14, Fax: +32 92643575
}

\begin{abstract}
In case of fire in an atrium, a smoke and heat control (SHC) system can be designed to improve safety inside the atrium. An important design criterion is the smoke free height in the atrium. This smoke free height is the result of a number of parameters, of which the fire heat release rate (HRR), the SHC extraction mass flow rate, the position of the extraction device or opening and the size and position of openings for make-up air are of primary importance. In the present paper, an extensive numerical study, based on Computational Fluid Dynamics (CFD), is presented in which these parameters are varied. The presence of a downstand or balcony is not covered in the study at hand. From the results, a correlation is presented for adhered spill plumes in atria without downstand, relating the smoke extraction rate to the smoke free height in the atrium. The smoke layer in the atrium is illustrated to become multidimensional beyond a certain threshold value of the smoke extraction rate and existing correlations, which do not take this phenomenon into account, are not conservative. The position and exact size of the make-up air inlet openings are shown not to affect the observations, as long as the openings are sufficiently large.
\end{abstract}

Key Words: atria; fire; smoke extraction; CFD; adhered spill plume 


\section{Highlights}

- CFD simulations are used as 'numerical experiments' for parameter variation

- a new formula is proposed for fire smoke extraction for adhered spill plumes in atria for higher extraction rates than studied so far

- impact of atrium height, width, length and fire heat release rate are included in the new formula

- the size of the make-up air inlet opening can have significant effect on smoke extraction in atria 


\section{Introduction}

Atria have become an increasingly popular type of architectural structure, e.g. in shopping malls, hotels or office buildings. In case of fire, a smoke and heat control (SHC) system can be an effective tool to improve safety for occupants or firemen inside the atrium. One of the most important criteria in the design of these SHC systems is the smoke free height in the atrium. Many experimental and numerical studies have already been performed in this context [1-9].

This smoke free height depends on a number of parameters. The fire heat release rate (HRR), the SHC extraction mass flow rate, the position of the extraction device or opening and the size and position of openings for make-up air are considered of primary importance. In this paper, an extensive parameter variation study is presented. The presence of a downstand or balcony, however, is not covered in the present paper.

Results from CFD simulations, obtained with FDS (Fire Dynamics Simulator, version 5.2.5 [10-11]), are used as 'numerical experiments'. The justification for this approach has already been proved in earlier work [12] and thus is not repeated here.

The objective of the study at hand is to develop a new correlation and to discuss flow field phenomena as observed from the CFD simulation results. 


\section{Setup}

The reduced-scale experimental atrium setup from Poreh et. al [8] is used as the setup for the study at hand, because experiments have been carried out for a range of conditions. The fire is located in the adjacent room to the atrium (Fig. 1) and four different convective HRRs $\left(\dot{Q}_{\text {conv }}\right)$ have been studied. For each HRR, the SHC extraction mass flow rate $(\dot{m})$ has been varied in the atrium ceiling, resulting in different smoke free heights above the spill edge $\left(z_{s}\right)$ in the atrium. The mass flow rate at the spill edge $\left(\dot{m}_{s}\right)$ and depth of the emerging smoke layer $\left(d_{s}\right)$ have been measured.

From the set of experiments, Poreh et al. proposed a single formula [8] to calculate the required extraction mass flow rate to ensure a certain smoke free height in the atrium. In recent work, an experimental study of the 2D adhered spill plume by Harrison and Spearpoint [9], including a variation of the atrium width $W_{s}$, the results of Poreh et. al [8] have been confirmed and the following expression was suggested:

$\dot{m}\left(z_{s}\right)=0.08 W_{s}^{2 / 3} \dot{Q}_{\text {conv }}^{1 / 3}\left(z_{s}+d_{s}\right)+\dot{m}_{s}$.

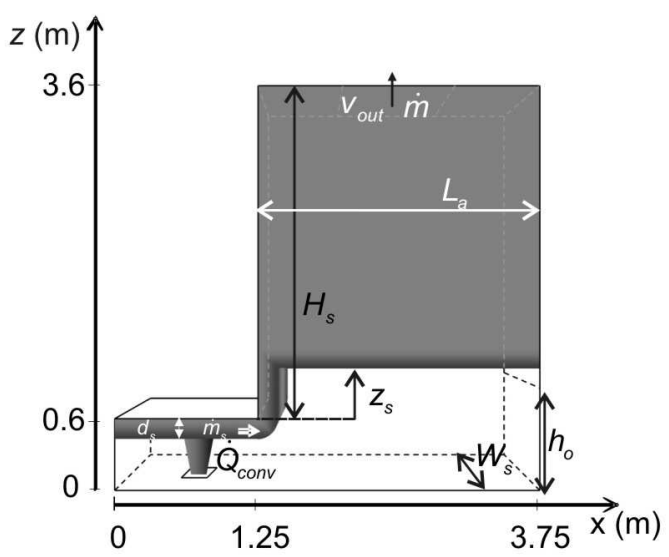

Figure 1. Atrium and adhered spill plume, basic configuration.

The experiments of Poreh et. al [8] have been reproduced with CFD simulations on grids with cubic cells with edge size $2.5 \mathrm{~cm}$ in [12]. Here, the CFD calculations as carried out with FDS 
(Fire Dynamics Simulator, version 5.2.5 [10-11]) are performed on grids with cubic cells of edge size $5 \mathrm{~cm}$. All other settings are identical as in [12]. In particular, unless mentioned otherwise, at the outlet opening in the ceiling of the atrium, a constant velocity $v_{\text {out }}$ is imposed for the extraction of smoke from the atrium. As the grid contains fewer cells, the calculation cost of the simulations is significantly reduced. As in [12], the smoke layer interface temperature is calculated with the $\mathrm{N}$-percent rule with $\mathrm{N}=30: T_{\text {int }}=T_{0}+\left(T_{\max }-T_{0}\right) \mathrm{N} / 100$. From the interface temperature, the smoke layer interface height above the spill edge $\left(z_{s}\right)$ is calculated. The values for $d_{s}, \dot{m}\left(z_{s}\right), \dot{m}_{s}$ and $z_{0}$ are calculated from the simulation output data as explained in [12]. Figure 2 confirms that the mesh is still sufficiently fine for the sake of the present paper.

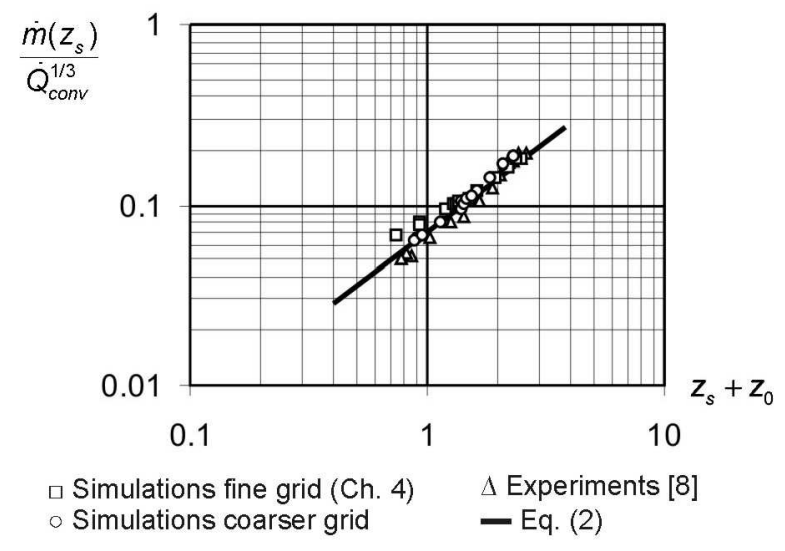

Figure 2. Confirmation that the 'coarser' grid (used in the present paper) is sufficiently fine for the sake of the present paper. Simulation results for the 'basic' setup. 


\section{Parameter Variation - Presentation of Results}

First, the SHC extraction mass flow rate is varied beyond the range studied in $[8,9,12]$ in the basic configuration (Fig. 1). Both mechanical and natural ventilation are examined.

Next, starting from this basic configuration, variations of fire heat release rate, atrium width and atrium height above the spill edge are performed. A new relation for the entire smoke extraction rate range is also presented.

Finally, variation of some of the ventilation parameters is discussed: position of the smoke extraction opening and size and position of the makeup air inlet opening.

The results are presented as graphs for the smoke layer interface height $z_{s}$ as function of the SHC extraction mass flow rate $\dot{m}\left(z_{s}\right)$. Eq. (1) is recast as:

$\dot{m}\left(z_{s}\right)=\left(0.08 W_{s}^{2 / 3} \dot{Q}_{\text {conv }}^{1 / 3}\right) z_{s}+\left(0.08 W_{s}^{2 / 3} \dot{Q}_{\text {conv }}^{1 / 3} d_{s}+\dot{m}_{s}\right)$

For each combination of input variables studied, the input values and the values for $\dot{m}_{s}$ and $d_{s}$ as obtained from the simulations, are inserted. E.g., in the basic configuration (Fig. 1) with HRR $\dot{Q}_{\text {conv }}=11.9 \mathrm{~kW}$ and atrium width $W_{s}=0.9 \mathrm{~m}([8,9])$, the values for smoke layer depth and mass flow rate emerging from the adjacent room are $d_{s}=0.17 \mathrm{~m}$ and $\dot{m}_{s}=0.09 \mathrm{~kg} / \mathrm{s}$ in the simulations and thus Eq. (2) becomes:

$\dot{m}\left(z_{s}\right)=0.17 z_{s}+0.119$.

As the atrium height is $3.6 \mathrm{~m}$ and the spill edge is at height $0.6 \mathrm{~m}$, application of Eq. (3) reveals that the maximum possible value to be obtained for the smoke free height above the spill edge is $z_{s}=3 \mathrm{~m}$, corresponding to an extraction mass flow rate of $0.63 \mathrm{~kg} / \mathrm{s}$. Yet, higher extraction mass flow rates are also studied in the present paper. 


\section{Discussion of Results}

\subsection{Variation of Smoke Extraction Rate (Mechanical Ventilation)}

The basic configuration concerns a fire HRR $\dot{Q}_{\text {conv }}=11.9 \mathrm{~kW}$ and atrium width $W_{s}=0.9 \mathrm{~m}$. Within this configuration, the extraction mass flow rate is varied, beyond the range studied in $[8,9,12]$. This is relevant since the simulations reveal that, as the extraction mass flow rate increases beyond a certain value, the smoke layer becomes multi-dimensional, meaning that the smoke interface height is not uniform in the atrium (Fig. 3). This effect is also related to the limited atrium size and the central position of the extraction outlet (see below).

The explanation is as follows. The smoke rises along the left vertical wall and as it is deflected underneath the atrium ceiling, it has momentum in the $x$-direction. If this momentum becomes too high, not all the smoke can be extracted by the mechanical smoke extraction outlet (in the $z$-direction). The non-extracted smoke creates a large-scale vortex in the atrium and thus causes the multi-dimensional effect.
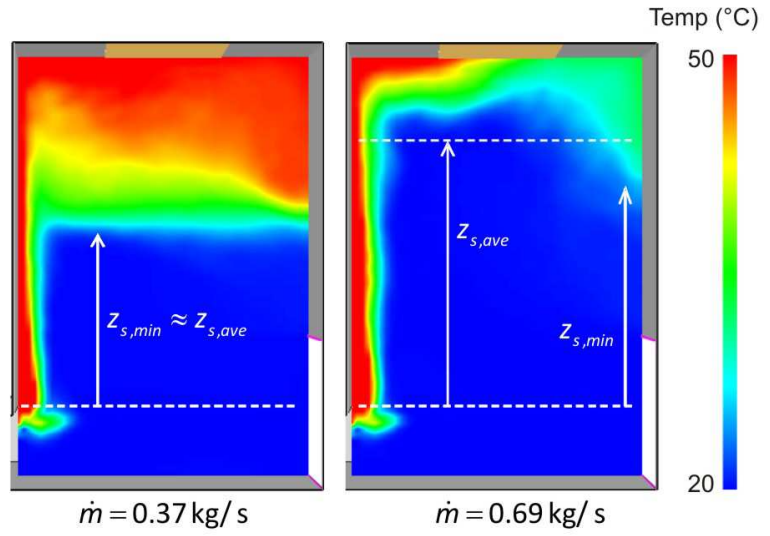

Figure 3. One-dimensional (left) versus multi-dimensional (right) smoke layer pattern in atrium. Temperature fields at $\mathrm{y}=0.45 \mathrm{~m}$, time-averaged statistically steady data (averaging period of $20 \mathrm{~s}$ ). 
In such a smoke layer pattern, it is not straightforward to define an unambiguous value for smoke free height. In the remainder of this paper, both the minimum and average values of the smoke free height in the atrium are shown in the presentation of the results. As long as these minimum and average smoke free heights have the same value, the smoke layer is onedimensional. When the minimum value becomes lower than the average one, this reflects a multi-dimensional pattern.

Fig. 4 shows the effect of the variation of the extraction mass flow rate. The left panel shows results when the outlet velocity $v_{\text {out }}$ at the extraction outlet is varied between $0.2 \mathrm{~m} / \mathrm{s}$ and 0.9 $\mathrm{m} / \mathrm{s}$, while maintaining the outlet opening area constant at $0.9 \mathrm{~m}^{2}$. The graph clearly shows that the results of both experiments and simulations are in very good agreement with Eq. (1) for extraction mass flow rates below $0.4 \mathrm{~kg} / \mathrm{s}$. Within this range, the smoke layer pattern is essentially one-dimensional. When the extraction mass flow rate exceeds $0.4 \mathrm{~kg} / \mathrm{s}$, however, the smoke layer becomes multi-dimensional and the simulation results deviate from Eq. (1). The average smoke layer interface height follows a new slope in the graph, starting from a certain threshold value. From that point onward, the smoke layer height still increases with increasing mass flow rate, but slower than is the case for lower extraction mass flow rates below the threshold value. The vortex at the right-hand side of the atrium (Fig. 3) causes a much lower smoke-free height than what would be expected from extrapolation of the onedimensional smoke layer results. The dashed line already indicates the resulting relation presented below, Eq. (11).

For now, this relation can be presented as

$$
\dot{m}\left(z_{s}\right)=a z_{s}+b .
$$

The smoke layer shows the multi-dimensional effect in Fig. 4 starting from the value $z_{s} \approx 2 \mathrm{~m}$, i.e.:

$z_{s, \text { threshold }}=2 / 3 H_{s}$. 

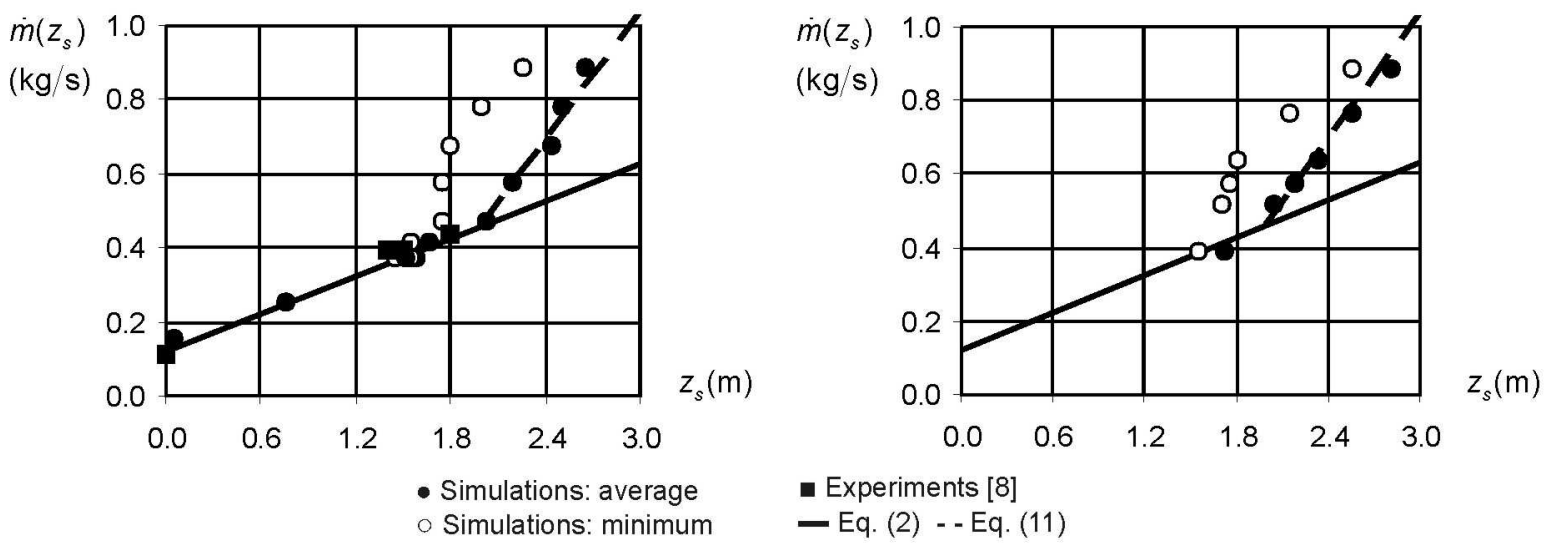

Figure 4. Basic configuration simulation results. Smoke extraction with mechanical ventilation. Left: $v_{\text {out }}$ varies $(0.2 \mathrm{~m} / \mathrm{s}$ to $0.9 \mathrm{~m} / \mathrm{s})$, constant outlet opening area $\left(0.9 \mathrm{~m}^{2}\right)$. Right: constant $v_{\text {out }}(0.6 \mathrm{~m} / \mathrm{s})$, outlet opening area varies $\left(0.49 \mathrm{~m}^{2}\right.$ to $\left.1.35 \mathrm{~m}^{2}\right) . \dot{Q}_{\text {conv }}=11.9 \mathrm{~kW}$. Solid line: Eq. (1); dashed line: Eq. (11).

With mechanical ventilation, the SHC extraction mass flow rate at the ceiling of the atrium can also be altered by keeping the extraction velocity $v_{\text {out }}$ fixed and altering the outlet opening area. The right panel of Fig. 4 shows results (with $v_{\text {out }}=0.6 \mathrm{~m} / \mathrm{s}$ ). For the average smoke layer height, the results are practically identical to what is shown in the left panel of Fig. 4. Thus, the smoke layer height $z_{s, a v}$ depends on the value of the SHC extraction mass flow rate $\dot{m}\left(z_{s}\right)$, not on the way in which $\dot{m}\left(z_{s}\right)$ is imposed. Small differences are observed in the evolution of the minimum smoke layer height $z_{s, m i n}$, but clearly the primary factor is the value of $\dot{m}\left(z_{s}\right)$, not the way in which $\dot{m}\left(z_{s}\right)$ is imposed. For completeness, it is worth mentioning that the deviation from the solid line is not a plug-holing effect: even with the highest values for $\dot{m}\left(z_{s}\right)$, the entire outlet opening area is extracting smoke (also in the right panel of Fig. 4).

\subsection{Variation of Smoke Extraction Rate (Natural Ventilation)}

Smoke can also be extracted from the atrium by means of natural ventilation. In this situation, the extraction opening area is varied, but no extraction velocity is imposed. The ventilation 
opening area is increased in the simulations until the situation is reached where the opening is so large that make-up air starts to flow through this opening into the atrium. Such a situation is considered beyond the scope of the present study and as a consequence, the range of values considered is less wide than in section 4.1. The extraction opening area is increased here from $0.49 \mathrm{~m}^{2}$ to $1.35 \mathrm{~m}^{2}$.

The results are displayed in Fig. 5. While the range of values considered is more limited than in Fig. 4, the results for $z_{s, a v}$ again match the dashed line well and also the results for $z_{s . m i n}$ are very similar to what was presented in Fig. 4. Above the threshold value, the multidimensional smoke layer situation again appears. Until the situation is reached where makeup air enters the atrium through the opening in the ceiling, the evolution of the SHC extraction mass flow rate with smoke layer height essentially does not depend on the type of ventilation, mechanical or natural.
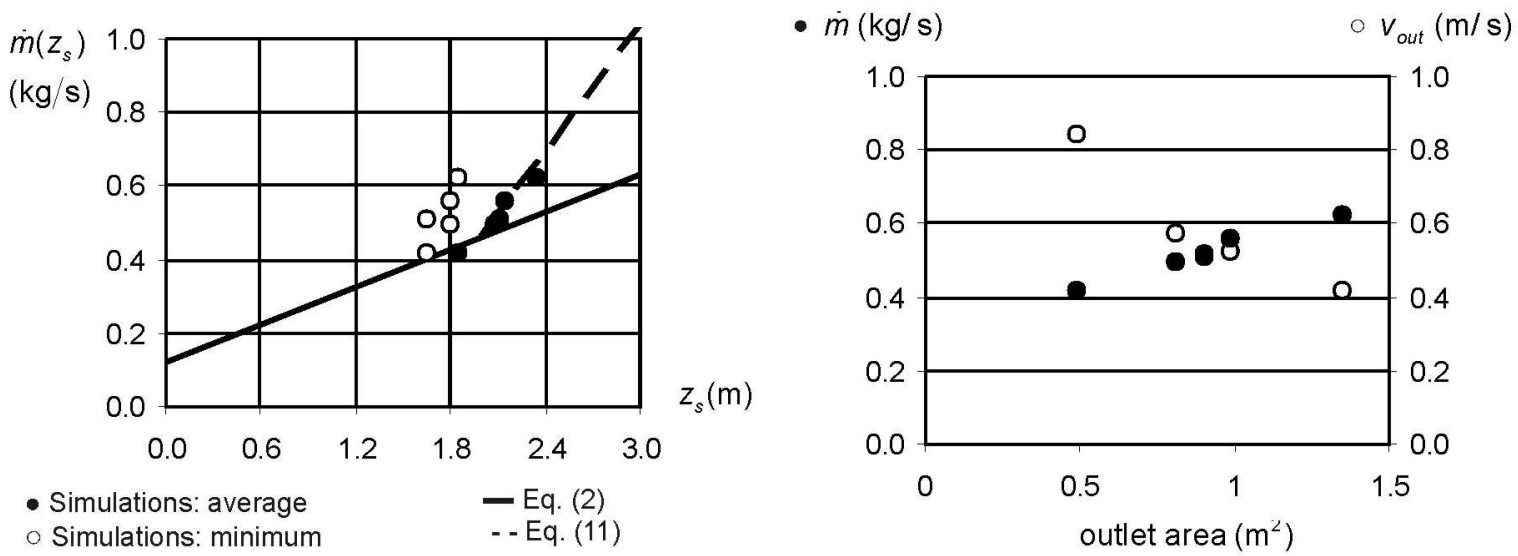

Figure 5. Smoke extraction by means of natural ventilation. Left: evolution of $\dot{m}\left(z_{s}\right)$ as function of $z_{s}$. Solid line: Eq. (2); dashed line: Eq. (11). Right: evolution of $\dot{m}\left(z_{s}\right)$ and $v_{\text {out }}$ as function of outlet opening area.

The right panel of Fig. 5 shows the dependence of the SHC extraction mass flow rate and $v_{\text {out }}$ on the outlet opening area. Although $v_{\text {out }}$ decreases with increasing outlet opening area, the 
SHC extraction mass flow rate increases. For an outlet area of $0.9 \mathrm{~m}^{2}$, the outlet velocity in the case of natural ventilation is $v_{\text {out }} \approx 0.6 \mathrm{~m} / \mathrm{s}$, which is the same as in Fig. 4 for mechanical ventilation.

\subsection{Variation of Fire HRR}

The question is addressed as to what extent the conclusions of the previous sections remain true for other fire HRRs. The HRR values as studied in [1,2] are considered and, as in the previous sections, the SHC extraction mass flow rate is varied beyond the range studied previously. Since the dependence on the way the SHC extraction mass flow rate is imposed is small (see sections 4.1 and 4.2), only mechanical ventilation is considered from now on, with fixed outlet opening area $\left(0.9 \mathrm{~m}^{2}\right)$ and variable outlet velocity $v_{\text {out }}$.
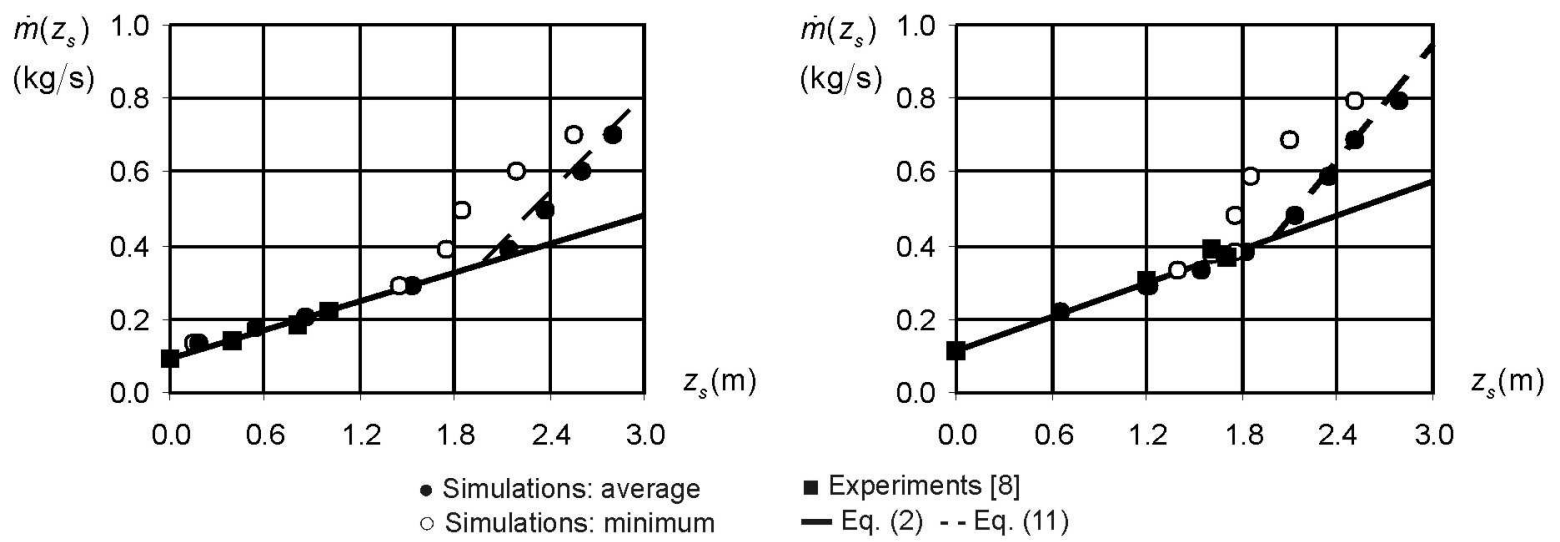

Figure 6. Basic configuration simulation results. Smoke extraction with mechanical ventilation. Left: $\dot{Q}_{c o n v}=5.4 \mathrm{~kW}$. Right: $\dot{Q}_{\text {conv }}=8.8 \mathrm{~kW}$. Solid line: Eq. (2); dashed line: Eq.

Fig. 6 reveals the results, to be compared to Fig. 4 (left). The results basically confirm the change of slope in the increase in smoke layer height with increasing SHC extraction mass flow rate beyond a certain threshold value. As in Fig. 4, this threshold value is at $z_{s}=2 / 3 H_{s}$ (Eq. (5)), indicating that this value is essentially independent of the fire HRR, for the range studied. The slope of the dashed lines (Eq. (4)) is found to vary as: 
$a \sim \dot{Q}_{\text {conv }}^{1 / 3}$.

This is in line with Eq. (2).

It is interesting to observe that the threshold value $\left(z_{s, \text { threshold }}=2 / 3 H_{s}\right.$ in the geometry at hand) is primarily determined by the geometric lay-out, and not by the fire heat release rate. A possible explanation is that the smoke dynamics is affected by both buoyancy and forced flow phenomena. Indeed, increasing the extraction rate implies increased intake air flow rates. The resulting flow inside the atrium then directly depends on the geometric lay-out (of the atrium and the ventilation openings). In the geometry studied, with the intake air opening in the opposite side of the room with the fire source, a large clockwise vortex structure is generated (see below, section 4.7). When the extraction rate is high enough (i.e. so high that the threshold value $z_{s, \text { threshold }}$ is exceeded) the multi-dimensionality appears. This is essentially due to the strong upward momentum of the smoke plume, which is not only driven by buoyancy, but which is also interacting with the (forced) flow field as generated by the intake air. This probably explains why the threshold value is primarily determined by the geometric lay-out, not so much by the fire heat release rate. This is discussed to a further extent in section 4.7.

\subsection{Variation of the atrium width}

Keeping the fire HRR fixed to $\dot{Q}_{\text {conv }}=11.9 \mathrm{~kW}$, the atrium width is varied. Starting from the basic configuration $\left(W_{s}=0.9 \mathrm{~m}\right)$, the width is increased $\left(W_{s}=1.2 \mathrm{~m}\right)$ and decreased $\left(W_{s}=\right.$ $0.75 \mathrm{~m}$ and $0.6 \mathrm{~m}$ ). The results are shown in Fig. 7. The threshold value for the change in slope and the formation of the multi-dimensional smoke layer is confirmed to be $z_{s}=2 / 3 H_{s}$ (Eq. (5)). The slope of the dashed lines varies as:

$a \sim W_{s}^{2 / 3}$.

This is again in line with Eq. (2). 
In order to confirm the effects of changing fire HRR and the atrium width together, a set of simulations has been performed with $W_{s}=0.6 \mathrm{~m}$ and $\dot{Q}_{\text {conv }}=5.4 \mathrm{~kW}$. Expressions (5), (6) and (7) have been confirmed (not shown here).
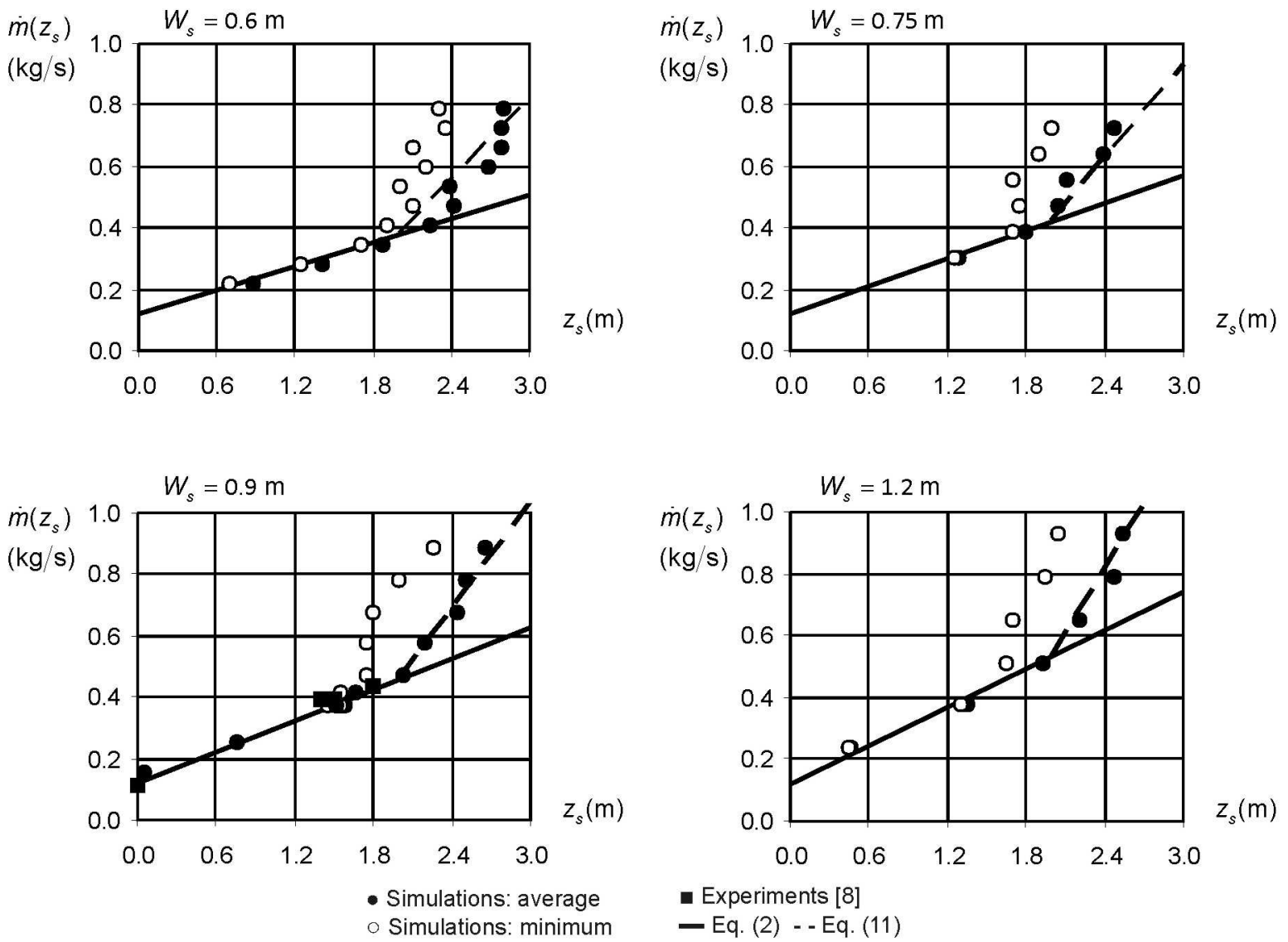

Figure 7. Impact of atrium width. Smoke extraction with mechanical ventilation.

$\dot{Q}_{\text {conv }}=11.9 \mathrm{~kW}$. Solid line: Eq. (2); dashed line: Eq. (11).

\subsection{Variation of the atrium height and length}

In the previous sections, the threshold value for the onset of the multi-dimensional smoke layer is $z_{s}=2 / 3 H_{s}$. A variation of $H_{s}$ is performed in order to verify whether this value is general. Figure 8 summarises the results for $H_{s}=2.4 \mathrm{~m}, 3.0 \mathrm{~m}, 4.2 \mathrm{~m}$ and $5.4 \mathrm{~m}$.

First of all, expression (5) for the threshold value is confirmed. Secondly, the slope of the dashed lines clearly depends on the value of $H_{s}$. Putting the results on a logarithmic scale, the dependence is with a negative exponent $(-5 / 3)$ : 
$a \sim H_{s}^{-5 / 3}$.

Further research is required to confirm or modify this exponent. Not surprisingly, the results evolve to Eq. (2) as $H_{s}$ increases: the evolution towards a multi-dimensional smoke layer, which causes the deviation from Eq. (2), will be less pronounced or less influential as the rise height itself increases. Indeed, the momentum in the x-direction upon deflection underneath the atrium ceiling decreases when the rising plume becomes cooler and less strong.
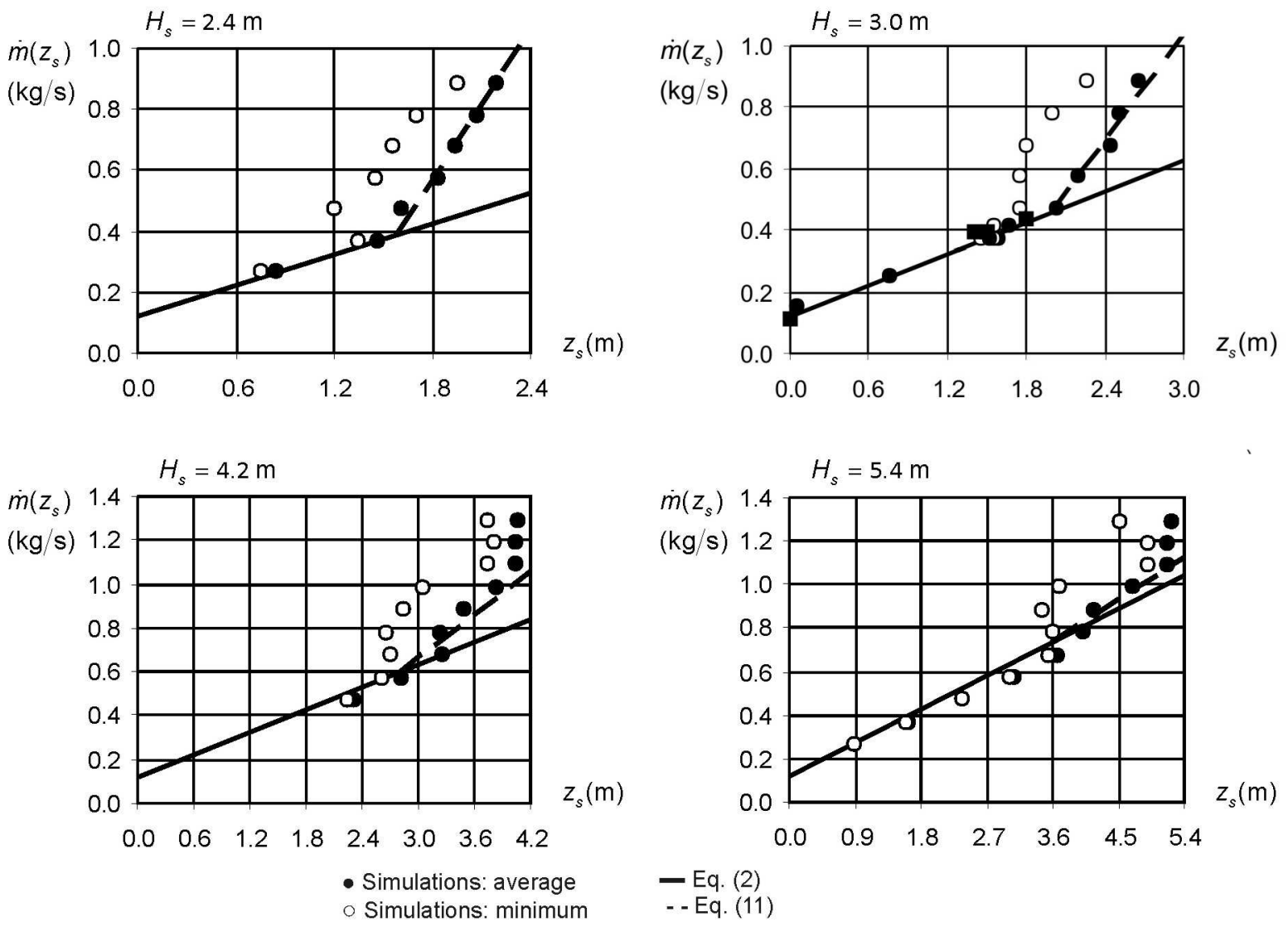

Figure 8. Impact of atrium height. Smoke extraction with mechanical ventilation.

$$
\dot{Q}_{\text {conv }}=11.9 \mathrm{~kW} \text {. Solid line: Eq. (2); dashed line: Eq. (11). }
$$

In this sense, a similar, but opposite, effect can be expected when the atrium length $L_{a}$ (see Fig. 1) is altered: the multi-dimensionality can be 'blocked' if the atrium becomes 'short' (in the x-direction). Fig. 9 confirms this (showing results for $L_{a}=1.25 \mathrm{~m}, 2 \mathrm{~m}, 3 \mathrm{~m}$ and $3.75 \mathrm{~m}$, 
with $L_{a}=2.5 \mathrm{~m}$ in the basic configuration). Similar results are obtained in atria with smaller length as in higher atria.
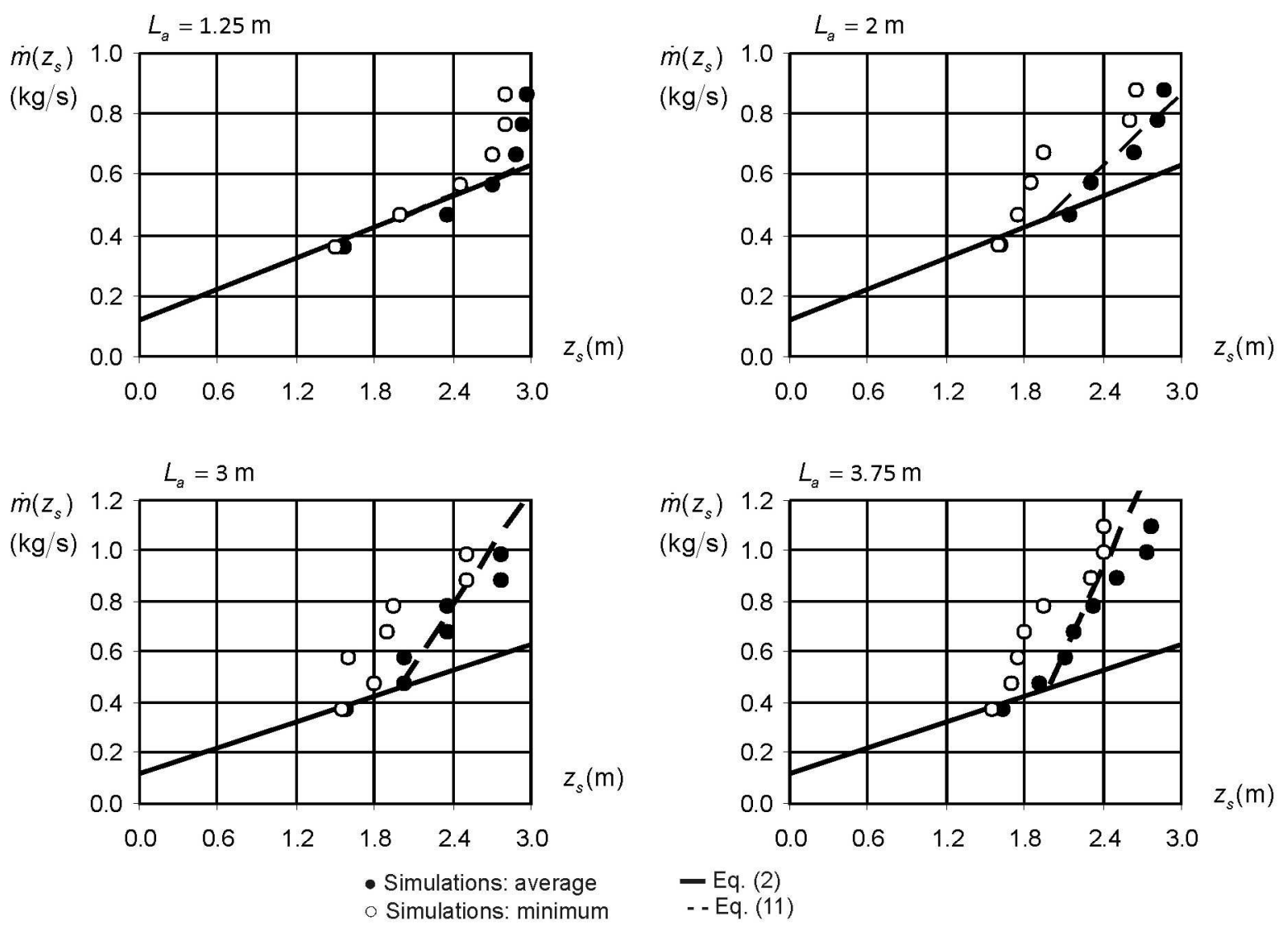

Figure 9. Impact of atrium length. Smoke extraction with mechanical ventilation.

$\dot{Q}_{\text {conv }}=11.9 \mathrm{~kW}$. Solid line: Eq. (2); dashed line: Eq. (11).

Also the slope change is similar:

$a \sim L_{a}^{5 / 3}$.

Again, further research is required to confirm or modify this exponent.

The deviation from Eq. (2) becomes small for high values of $H_{s} / L_{a}$ (see bottom right Fig. 8 and top left Fig. 9). Therefore, the results of this section can be summarised as:

$a \sim\left(\frac{L}{H_{s}}\right)^{5 / 3}$ for $H_{s} / L_{a}<2.5$ 
with expression Eq. (5) for the threshold value for the deviation from Eq. (2) still valid.

\subsection{Summary - New Correlation}

The results presented above are summarised in a new correlation for the configuration studied. This correlation is such that Eq. (2) is applied for one-dimensional smoke layers (i.e. $z_{s}<z_{s, \text { threshold }}=2 / 3 H_{s}$, Eq. (5), or $H_{s} / L_{a}>2.5$ ), while Eq. (4) is applied (with Eqs. (6), (7) and (10)) in multi-dimensional smoke pattern situations. The correlation is such that one single value is obtained for $z_{s}=2 / 3 H_{s}$. The result is:

$$
\left\{\begin{aligned}
\dot{m}\left(z_{s}\right)-\dot{m}_{s}=0.08 W_{s}^{2 / 3} \dot{Q}_{\text {conv }}^{1 / 3}\left(z_{s}+d_{s}\right) & \\
& \text { for } z_{s}<\frac{2}{3} H_{s} \text { or } \frac{H_{s}}{L_{a}}>2.5 \\
\dot{m}\left(z_{s}\right)-\dot{m}_{s}=0.08 W_{s}^{2 / 3} \dot{Q}_{\text {conv }}^{1 / 3}\left(2.5^{5 / 3}\left(\frac{L_{a}}{H_{s}}\right)^{5 / 3}\left(z_{s}-\frac{2}{3} H_{s}\right)+\frac{2}{3} H_{s}+d_{s}\right) & \text { for } z_{s}>\frac{2}{3} H_{s} \text { and } \frac{H_{s}}{L_{a}}<2.5
\end{aligned}\right.
$$

It is recalled that the presence of a balcony or downstand is not considered here. Fig. 10 shows the agreement of Eq. (11) with the values obtained in the CFD simulations.

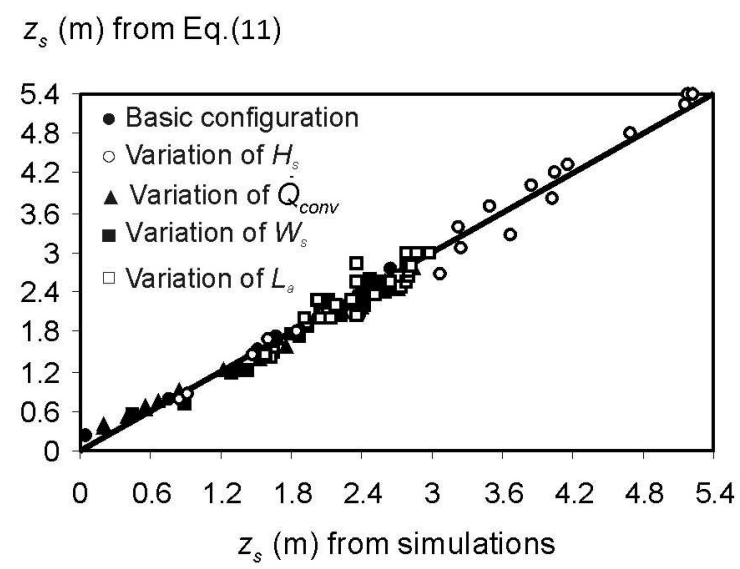

Figure 10. Agreement of correlation (11) to the CFD simulation results. 


\subsection{Variation of ventilation parameters}

Correlation (11) has been developed for an atrium configuration with the smoke extraction opening in the centre of the atrium ceiling, and the make-up air inlet opening in the wall opposed to the adjacent room where the fire occurs (Fig. 1). However, other ventilation configurations are possible in real-life atria. Therefore, the effect of the following parameters is studied here:

- position of the smoke extraction opening,

- position of the make-up air inlet opening,

- size of the make-up air inlet opening.

The basic configuration sis considered ( $\dot{Q}_{\text {conv }}=11.9 \mathrm{~kW}, W_{s}=0.9 \mathrm{~m}, L_{a}=2.5 \mathrm{~m}, H_{s}=3 \mathrm{~m}$ ).

\subsubsection{Position of smoke extraction opening}

Fig. 11 shows the position of the extraction outlet in the three different configurations studied. The middle outlet configuration corresponds to the basic configuration (Fig. 1).

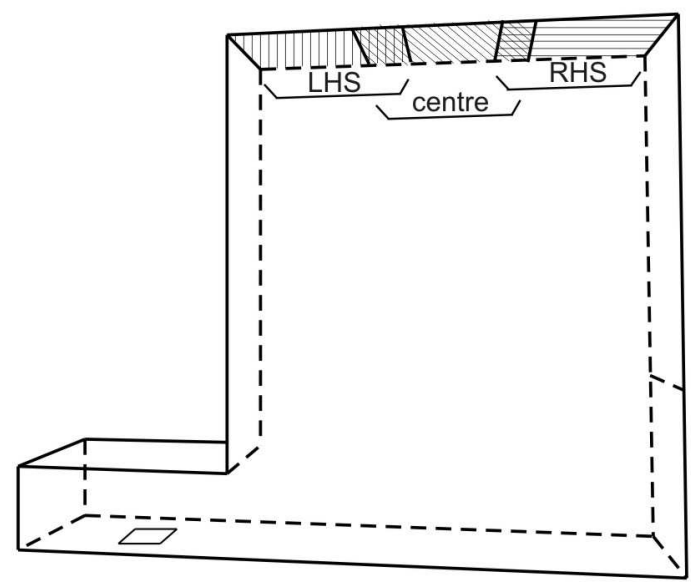

Figure 11. Different positions of smoke extraction opening considered.

The simulation results for the LHS and RHS positions are presented in Fig. 12, to be compared to Fig. 4 (left). In the LHS configuration, the deviation from the solid line (Eq. (2)) is clearly postponed, compared to the basic case (to which the dashed line corresponds). The 
reason is related to the explanation, given in section 4.1 , for the multi-dimensional smoke layer pattern. Indeed, as the extraction opening in the LHS configuration is placed directly above the rising adhered smoke plume, there is practically no horizontal momentum of the smoke layer near the outlet and the smoke can easily be extracted from the atrium. The results for the RHS configuration do not deviate much from the basic configuration. If anything, the change in slope is a little "worse": for the same extraction mass flow rate, the smoke layer interface height is lower in the atrium.
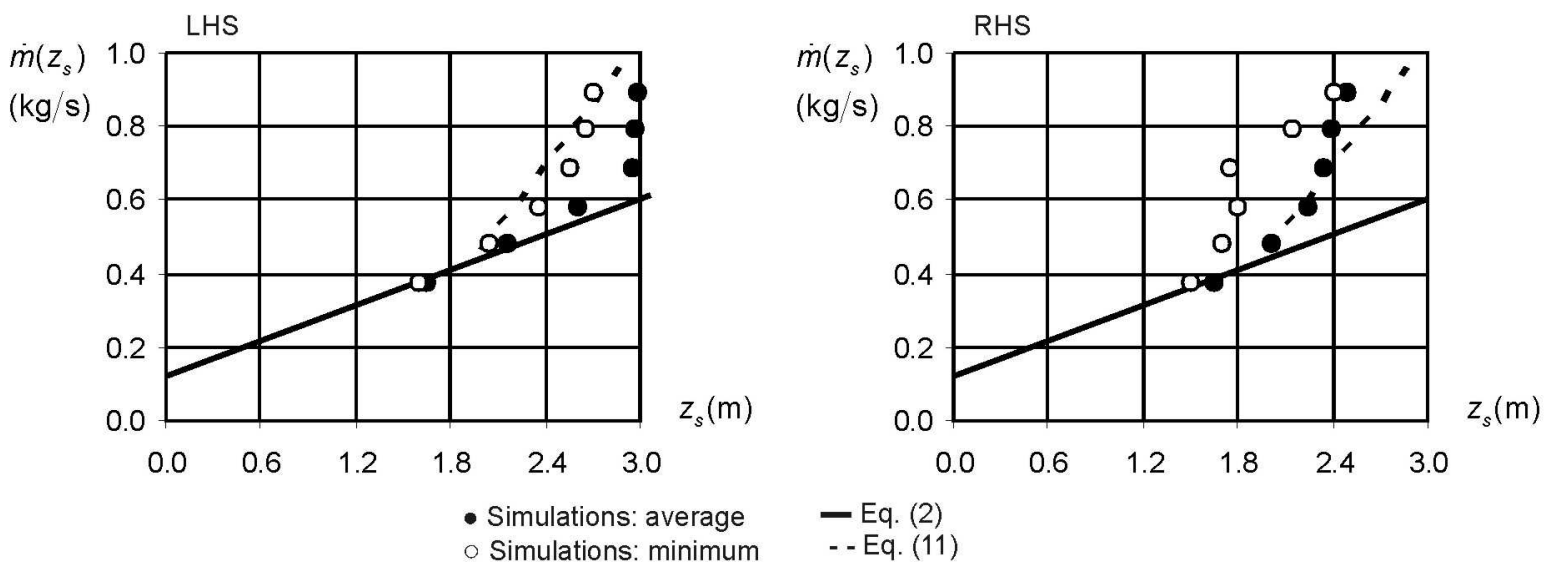

Figure 12. Impact of position of smoke extraction outlet opening. LHS, RHS: See Fig. 11. Solid line: Eq. (2); dashed line: Eq. (11).

Clearly, the LHS configuration results in the least stringent requirements in terms of required SHC smoke extraction mass flow rate. However, in reality the location of the fire is not known a priori, so that the LHS configuration cannot be relied upon. Therefore, applying correlation (2), in combination with the LHS configuration, cannot be seen as a conservative design approach. Bearing this in mind, the central position, applying correlation (11), can be considered as a conservative approach.

\subsubsection{Position of make-up air inlet}

Fig. 13 shows the atrium with the different positions of make-up air inlet openings considered. Position A corresponds to the basic configuration (Fig. 1). Positions B and C have 
been designed such that the smoke layer emerging from the adjacent room can turn around the spill edge, without smoke leaving the atrium through the inlet air openings. To that purpose, openings $\mathrm{B}$ and $\mathrm{C}$ do not cover the entire side of the atrium (they are only $2 \mathrm{~m}$ wide). A situation where smoke leaves the atrium through a make-up air inlet opening is beyond the scope of the present study.

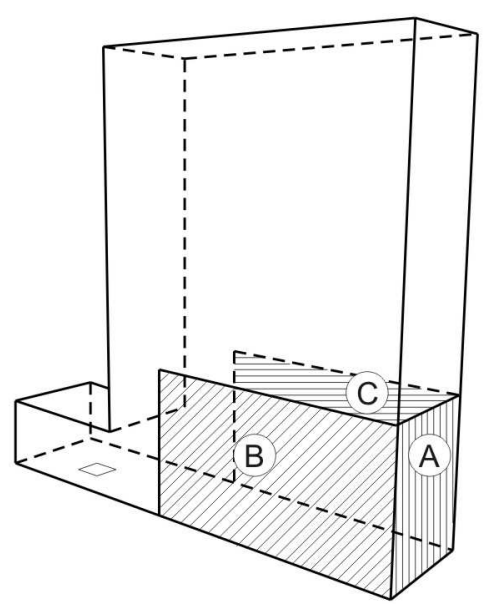

Figure 13. Different positions of the make-up air inlet opening considered.

Fig. 14 shows results for the basic situation (top left), as well as for the situations when openings $\mathrm{B}$ and $\mathrm{C}$ are open (top right), when all openings (A, B and C) are open (bottom left) and when only opening B is open (bottom right). When an inlet opening is present in the side wall, there is less tendency to form the multi-dimensional smoke layer pattern. This can be understood from Fig. 3: the clockwise vortex, stimulating the downward smoke motion at the right hand atrium wall, is less strong when the make-up air does not enter in line with this clockwise vortex. This explains the smaller differences between $z_{s, a v}$ and $z_{s . m i n}$ in the configurations with opening B than in the basic configuration. Also the deviation from Eq. (2) is postponed to higher $z_{s}$ values than given by Eq. (5). Yet, the differences between the simulation results for $z_{s, a v}$ and correlation (11) (which is the dashed line in Fig. 14) are not large and correlation (11) can be interpreted as conservative. Therefore, correlation (11) 
applies, independently of the position of the make-up air inlet opening (as long as this opening is sufficiently large, see next subsection).
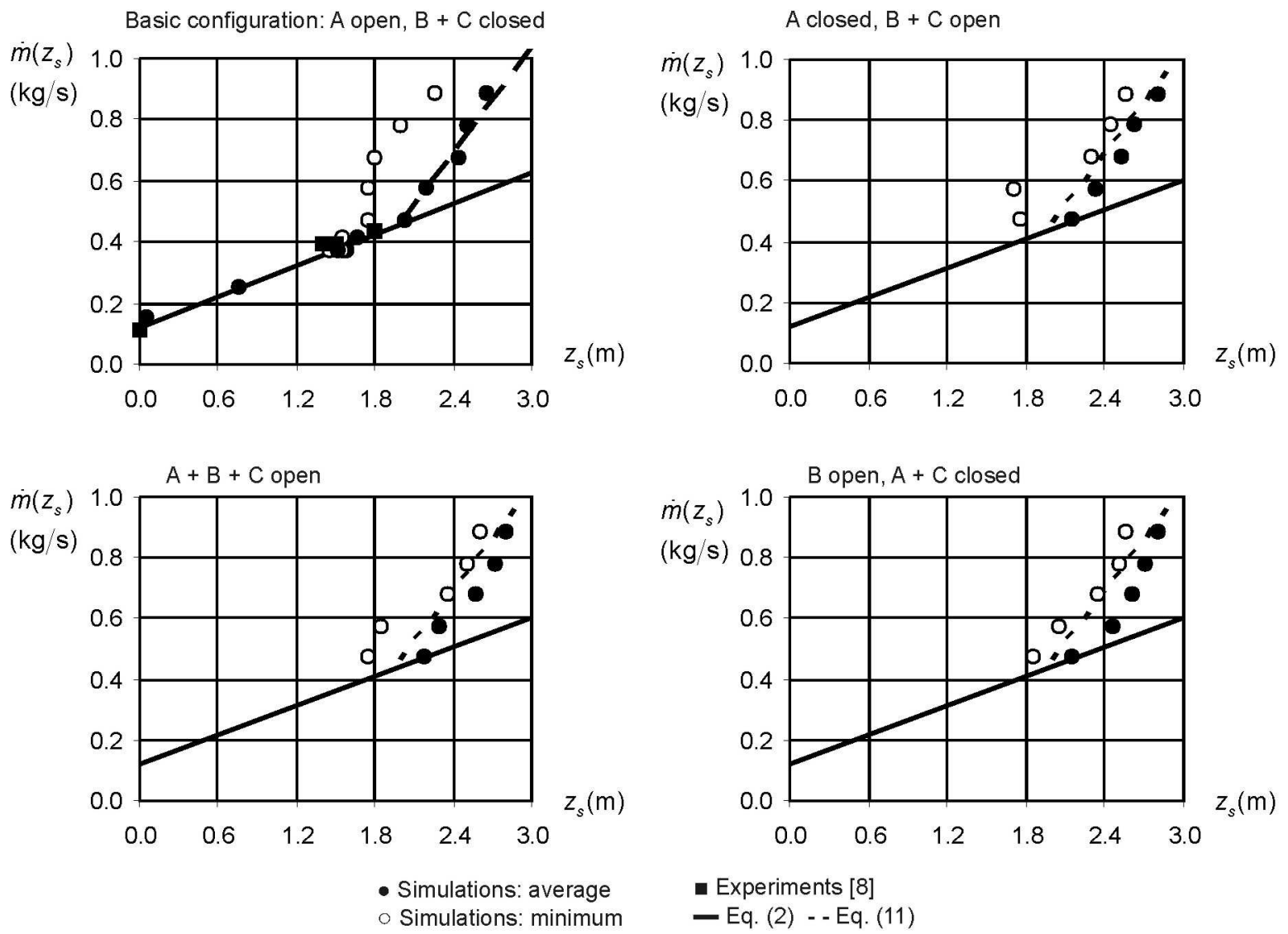

Figure 14. Impact of position of make-up air inlet opening. A, B and C: see Fig. 13. Solid line: Eq. (2); dashed line: Eq. (11).

For the configuration where only opening B is open, the possible effect of asymmetry in smoke patterns has been examined. For the cases at hand, this effect is negligible (not shown).

\subsubsection{Make-up air inlet opening size}

The final parameter studied in the present paper is the size of the make-up air inlet opening.

Configuration A is considered, with variation of the height $h_{o}$ of the opening (Fig. 1). 

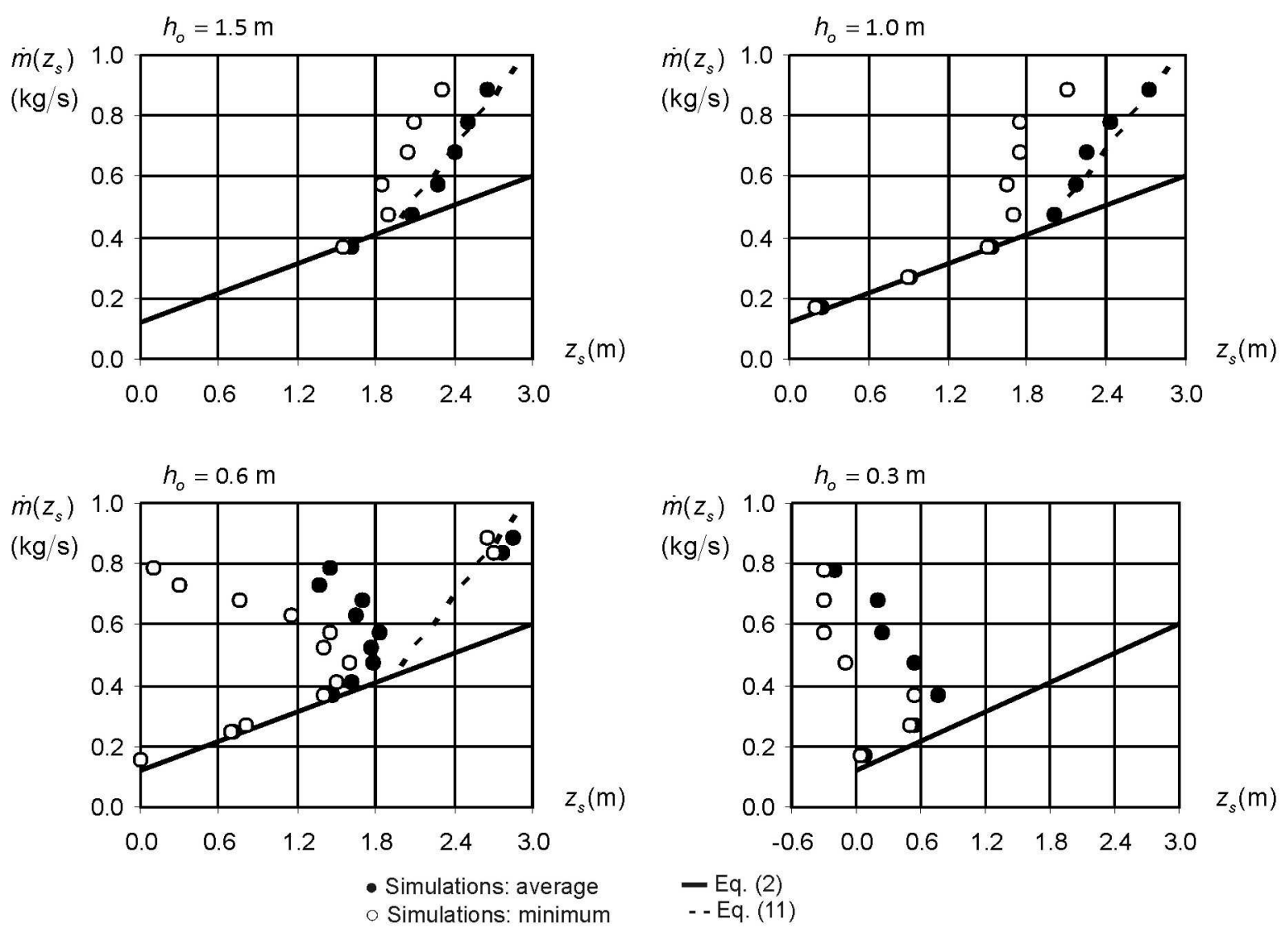

Figure 15. Impact of position of make-up air inlet opening height for Configuration A of Fig.

13. Basic configuration: $h_{o}=1.2 \mathrm{~m}$. Solid line: Eq. (2); dashed line: Eq. (11).

Figure 15 reveals that, as long as the make-up air inlet opening is sufficiently large, the results are hardly affected by the exact size of the opening. For the case at hand, the results match correlation (11), shown as dashed line, very well for $h_{o}>1.0 \mathrm{~m}$. For smaller inlet openings, on the other hand, the results strongly deviate from Eq. (11) and the deviation from Eq. (2) already occurs for lower values of $z_{s}$ : there is much more tendency towards a multidimensional smoke layer pattern due to a much stronger clockwise vortex, stimulated by the high velocity of the make-up air. This is visualised in Fig. 16. 


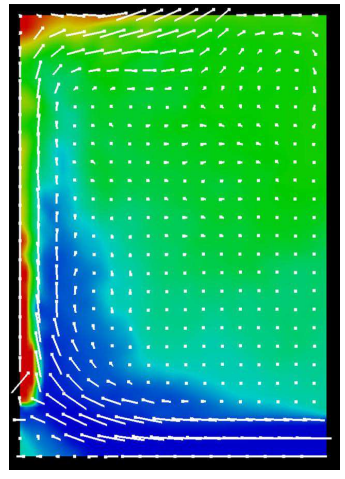

$h_{o}=0.3 \mathrm{~m}$

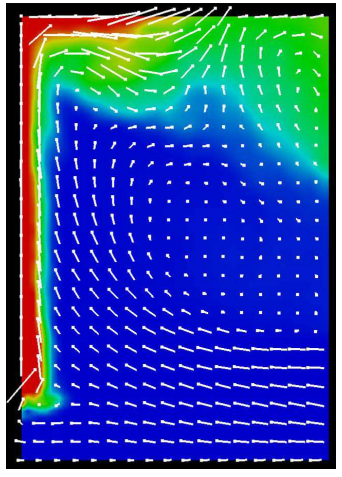

$h_{o}=1 \mathrm{~m}$

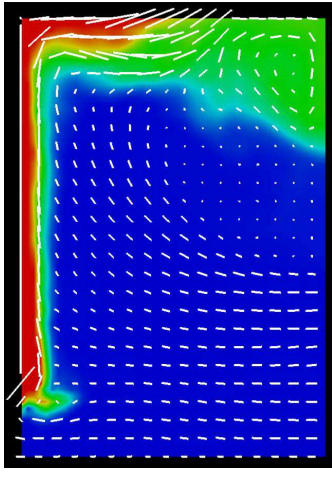

$h_{0}=1.5 \mathrm{~m}$

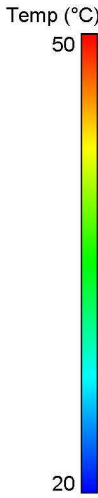

Figure 16. Temperature fields and velocity vectors in the vertical symmetry plane. Timeaveraged statistically steady results (averaging period of $20 \mathrm{~s}$ ).

These results demonstrate that the size of the inlet opening does not affect the smoke layer pattern as long as the opening is sufficiently large. When the opening is too small, the effectiveness of the SHC system is drastically reduced, not only due to possible pressure losses (which were not accounted for in the simulations, as a constant $v_{\text {out }}$ was imposed), but also due to changes in the flow field. Thus, it is extremely important to carefully consider make-up air inlet opening sizes during the design phase of an atrium building. 


\section{Conclusions}

In this paper, based on an extensive CFD simulation study in which several parameters have been varied, a correlation has been developed for 2D adhered spill plumes in atria without downstand, relating the smoke extraction mass flow rate to the smoke-free height in the atrium. The following conclusions prevail:

- When the SHC extraction mass flow rate exceeds a threshold value, the smoke layer in the atrium becomes multi-dimensional. This causes a deviation of the results from existing correlations (which do not consider this multi-dimensional situation). The existing correlations are not conservative.

- The threshold situation corresponds to a smoke rise height $z_{\text {s, threshold }}=2 / 3 H_{s}$, with $H_{s}$ the height difference between the spill edge and atrium ceiling.

- The multi-dimensional pattern can only occur if the atrium is not too high $\left(H_{s}<2.5 L_{a}\right.$ for the case studied), nor too short ( $L_{a}>0.4 H_{s}$ for the case studied).

- The observations do not depend on the type of smoke extraction (mechanical or natural ventilation).

- The position of the make-up air inlet openings does not strongly affect the observations, as long as they are sufficiently large.

- The exact size of the make-up air inlet openings does not affect the observations, as long as they are sufficiently large.

- The multi-dimensionality of the smoke layer pattern is related to the momentum of the smoke and the corresponding large-scale vortex. If the make-up air inlet openings are such that this vortex is stimulated, the effectiveness of the SHC system is reduced.

- The SHC system performs best when placed directly above the rising plume. However, as in most cases it is impossible to predict the exact location of the fire, it is suggested to put the smoke extraction opening in the middle of the atrium. 
- The correlation, Eq. (11), applicable only to the configuration studied, reads:

$$
\left\{\begin{aligned}
\dot{m}\left(z_{s}\right)-\dot{m}_{s}=0.08 W_{s}^{2 / 3} \dot{Q}_{\text {conv }}^{1 / 3}\left(z_{s}+d_{s}\right) & \\
& \text { for } z_{s}<\frac{2}{3} H_{s} \text { or } \frac{H_{s}}{L_{a}}>2.5 \\
\dot{m}\left(z_{s}\right)-\dot{m}_{s}=0.08 W_{s}^{2 / 3} \dot{Q}_{\text {conv }}^{1 / 3}\left(2.5^{5 / 3}\left(\frac{L_{a}}{H_{s}}\right)^{5 / 3}\left(z_{s}-\frac{2}{3} H_{s}\right)+\frac{2}{3} H_{s}+d_{s}\right) & \text { for } z_{s}>\frac{2}{3} H_{s} \text { and } \frac{H_{s}}{L_{a}}<2.5
\end{aligned}\right.
$$

Further research should lead to confirmation or modification of exponent 5/3.

\section{Acknowledgement}

Research funded by a Ph.D grant of the Institute for the Promotion of Innovation through Science and Technology in Flanders (IWT-Vlaanderen).

\section{References}

[1] Law, M., “A Note on Smoke Plumes from Fires in Multi-level Shopping Malls”, Fire Safety Journal 10(3): 197-202 (1986).

[2] Thomas, P.H., "On the Upward Movement of Smoke and Related Shopping Mall Problems”, Fire Safety Journal 12(3): 191-203 (1987).

[3] Law, M., "Measurements of Balcony Smoke Flow", Fire Safety Journal 24(2): 189195 (1995).

[4] NFPA 92B, Smoke Management Systems in Malls, Atria and Large Areas, National Fire Protection Association (2005).

[5] Poreh, M., Morgan, H.P., Marshall, N.R., Harrison, R., "Entrainment by TwoDimensional Spill Plumes”, Fire Safety Journal 30(1): 1-19 (1998).

[6] Thomas, P.H., Morgan, H.P., Marshall, N., “The Spill Plume in Smoke Control Design”, Fire Safety Journal 30(1): 21-46 (1998). 
[7] Harrison, R., Spearpoint, M., "Entrainment of air into a balcony spill plume", Journal of Fire Protection Engineering 16: 211-245 (2006).

[8] Poreh, M., Marshall, N.R., Regev, A., "Entrainment by adhered two-dimensional plumes", Fire Safety Journal 43: 344-350 (2008).

[9] Harrison R, Spearpoint M, Physical scale modeling of adhered spill plume entrainment, Fire Safety J 45: 149-158 (2010)

[10] McGrattan K, Hostikka S, Floyd J, Baum H, Rehm R, Mell W, McDermott R, Fire Dynamics Simulator (Version 5) Technical Reference Guide, NIST 1018-5, National Institute of Standards and Technology (2008)

[11] McGrattan K, Klein B, Hostikka S, Floyd J, Fire Dynamics Simulator (Version 5) User's Guide, NIST 1019-5, National Institute of Standards and Technology (2008)

[12] Tilley N, Rauwoens P, Merci B, Verification of the accuracy of CFD simulations in small-scale tunnel and atrium fire configurations, Fire Safety J. 46 (2011) 186-193 